\title{
The Magnetoresistance in Iron-based Superconductors
}

\author{
B. Lv ${ }^{1}$, R. B. Xie ${ }^{1}$, S. L. Liu ${ }^{2}$, G. J. Wu ${ }^{3}$, H. M. Shao ${ }^{1}$, and X. S. Wu ${ }^{1 *}$ \\ ${ }^{1}$ National Lab of Solid State Microstructures, Department of Physics, Nanjing University, Nanijng 210093, PR China \\ ${ }^{2}$ College of Mathematics and Physics, Nanjing University of Posts and Telecommunications, Nanjing 210003, PR China \\ ${ }^{3}$ College of Sciences, Nanjing University of Technology, Nanjing 210003, PR China
}

(Received 14 May 2011, Received in final form 11 June 2011, Accepted 12 June 2011)

\begin{abstract}
The phase transition of vortex matter from solid to liquid was studied in iron-based superconductors. Based on the traditional vortex glass theory, we have examined the magnetoresistivity data of iron-based superconductors using our extended thermal activation model: $\rho(B, T)=\rho\left(\left(T-T_{g}(B)\right) /\left(T_{c}(0)-T_{g}(B)\right)\right)^{v(z-1)}$. We predict that the magnetic field-dependent area $S+S_{0}$ which integrates $\rho$ with $\mathrm{T}$ is proportional to $B^{\beta}$, where $\beta$ is the vortex glass transition exponent. From our calculation, the vortex glass transition exponent is 0.33, close to the exponent of area $S_{0}+S$ is 0.31 in $\mathrm{SmO}_{0.9} \mathrm{~F}_{0.1} \mathrm{FeAs}$; the exponent of area $S$ is 0.63 , which is close to the irreversibility line exponent $2 / 3$. Both of the results show the validity of our model. In addition, our model is shown to be effective in describing irreversibility behavior in layered superconductors.
\end{abstract}

Keywords : superconductors, vortex glass, phase transition, irreversibility line

\section{Introduction}

Recently discovered iron-based superconductors have various attractive properties, such as their superconducting transition temperatures and upper critical magnetic fields are higher than those of $\mathrm{MgB}_{2}$ and comparable to those of the cuprate superconductors [1-5]. One of the interesting characteristics is that they exhibit a layered structure with conducting layers of FeAs and charge reservoir layers of $\mathrm{ReO}$. This layered structure is very similar to that of cuprate superconductors. Like cuprate superconductors [6-8], the phase transition of vortex matter from solid to liquid has also been studied in ironbased superconductors recently [9].

The vortex solid state is characterized by a nonzero critical current density, while the vortex liquid is dissipative at all currents. The solid-to-liquid phase transition is most likely a first order melting transition in very clean systems, but it turns into a second order vortex glass transition for highly disordered systems involving point defects or Bose glass transitions in systems with corrected defects like ion-induced columnar defects or twin boundaries. With the assumption of the coherence length

\footnotetext{
*Corresponding author: Tel: +86 2583594402

Fax: +86 25 83594402, e-mail: xswu@nju.edu.cn
}

$\xi_{g} \sim\left|T-T_{g}\right|^{-v}$ and the characteristic time $\tau_{g} \propto \xi_{g}^{z}$, the dc current-voltage $(I-V)$ characteristics for a vortex glass with quenched disorder are scaled with

$$
E \xi_{g}^{z+1} \propto F_{ \pm}\left(J \xi_{g}^{d-1}\right)
$$

where $d$ is the dimensionality, $E$ is the electrical field, $J$ is the current density, and $z$ is the dynamic critical exponent [10-13]. Rydh et al [6,7] modified the original vortex glass theory by introducing a new form of coherence length: $\xi_{g}(T) \sim\left|k_{B} T / U_{\text {eff }}-1\right|^{-v}$, with $U_{\text {eff }}$ effective pinning energy.

In this paper, we discuss the broadening of the resistive transition of iron-based superconductors and compared it with cuprate superconductors. From the method of scaling analysis, we find that the broadening of the resistive transition can be well described by our model.

\section{Model}

The broadening of the resistive transition of HTSC has been the subject of numerous experimental and theoretical studies. It is an intrinsic effect, since it was observed in polycrystalline and single crystal samples. Tinkham [14] suggested that the width of the transition $(\Delta T)$ is proportional to $B^{2 / 3}$ and indicated that this agrees well with that of the $\mathrm{YBCO}$ and $\mathrm{BiSrCaCuO}$ system. How- 
ever, Palstra et al. [15] have found that this flux creep model is only applicable in a limited temperature regime.

Here, we use our former model [8] to investigate the iron-based superconductors prepared by Chen [16] and other types of superconductors $[17,18]$.

In the thermal activation model, the resistance can be considered to be a result of the competition of thermal activation $k_{B} T$ and the effective pinning energy $U_{\text {eff }}$, this results in the formula:

$$
\rho(B, T)=\rho_{n}\left(\frac{T-T_{g}(B)}{T_{c}(0)-T_{g}(B)}\right)^{v(z-1)}
$$

where $\rho_{n}$ appears to take a value close to the normal state resistance (In this paper, the critical temperature $T_{c}(0)$ of the iron-based superconductors [16], $\mathrm{MgB}_{2}$ [18] and our samples [17] are referenced from the reported data.). This expression is a typical scaling function of critical phenomena and is in excellent agreement with the vortex glass phase transition theory.

We studied the validity of Eqs. (2) by the method of the integral of the magneto resistance curve. Then we defined the magneto resistance as [19]

$$
\Delta \rho=\rho(B, T)-\rho(0, T)
$$

It is obvious that the area enclosed by the $\Delta \rho$ curve and the zero level represents the total increase in dissipation caused by the field. It is also equal to the extra work done by the external current source when the field is applied.

The area $S$ that is calculated is

$$
S=\int_{T_{g}(B)}^{T_{c}(0)} \rho(B, T) d T-\int_{T_{g}(0)}^{T_{c}(0)} \rho(0, T) d T,
$$

From Eqs. (2), one directly obtains

$$
\begin{aligned}
S= & \rho_{n} \frac{1}{v(z-1)+1}\left(T_{c}(0)-T_{g}(B)\right) \\
& -\rho_{n} \frac{1}{v(z-1)+1}\left(T_{c}(0)-T_{g}(0)\right)
\end{aligned}
$$

Note that because of the external current source and the intrinsic quenched disorder, the critical temperature $T_{c}(0)$ is a little different from the glass transition temperature $T_{g}(0)$ even in a zero field. Though the difference is quite small, the magnitude of $\rho_{n} /[v(z-1)+1]$ is large enough that the second term at the right hand of Eqs. (5) should not be neglected.

From the relation $1-T_{g}(B) / T_{c}(0) \propto B^{\beta}$ [20] we have

$$
S+S_{0} \propto \rho_{n} \frac{T_{c}(0)}{v(z-1)+1} B^{\beta}
$$

where $S_{0}=\rho_{n}\left(T_{c}(0)-T_{g}(0)\right) /[v(z-1)+1]$.

The magnetic field dependence of the area $S$ predicted by Tinkham is proportional to $B^{2 / 3}$, and now we predict the area $S_{0}+S$ is proportional to $B^{\beta}$, in addition, we plot the $\log (S)$ versus $\log (B)$ to obtain the exponent $\lambda$, then compare it with the prediction of Tinkham. The numerical integration is handled by the Original 7.0 using the experimental data. In the end, we plot the scaling behavior of the raw data close to $T_{c}$ or $T_{g}$ to discuss the validity of our model.

\section{Result and Discussion}

We studied Eqs. (2) and (6) with 3 samples: $\mathrm{SmO}_{0.9} \mathrm{~F}_{0.1^{-}}$ FeAs [16], and our previous samples $\mathrm{Y}_{0.8} \mathrm{Er}_{0.2} \mathrm{Ba}_{2} \mathrm{Cu}_{3} \mathrm{O}_{7-\delta}$ [17] and $\mathrm{MgB}_{2}$ [18]. An easy way to confirm our model is to plot $\log (B)$ versus $\log \left(1-T_{g}(B) / T_{c}(0)\right)$ with a slope of $1 / \beta$ and $\log (B)$ versus $\log \left(S+S_{0}\right)$ with a slope of $1 / \beta_{s}$ respectively.

In Fig. 1, the $\rho \sim T$ curves of the $\mathrm{SmO}_{0.9} \mathrm{~F}_{0.1} \mathrm{FeAs}$ sample are shown. For a more reasonable discussion, we avoid using the $\rho \sim T$ data in large fields where the dimensional transition will take place.

As we can see, a field of 9 Tesla slightly depresses the transition temperature by $1.4 \mathrm{~K}$ in $\mathrm{SmO}_{0.9} \mathrm{~F}_{0.1} \mathrm{FeAs}$, so we consider the broadening without any shift of the end point at $T_{c}(B)$.

The scaling behavior of vortex glass temperature $T_{g}$ versus field $B$ is shown in Fig. 2. In this paper, all the vortex glass temperatures are estimated by applying the Vogel-Fulcher relation: $(\partial \ln \rho / \partial T)^{-1} \propto\left(T-T_{g}(B)\right) / v(z-1)$ to the resistive tails [21].

Utilizing these glass transition temperatures in different fields, we fit the raw data by our model which has been shown in Fig. 1. The dash line is the theoretical fitting obtained by reasonable parameters $\rho_{n}=0.6 \mathrm{~m} \Omega \cdot \mathrm{cm}, T_{c}(0)$ $=54 \mathrm{~K}$, and $v(z-1)=3.9 \pm 0.4$. This is an explicit result

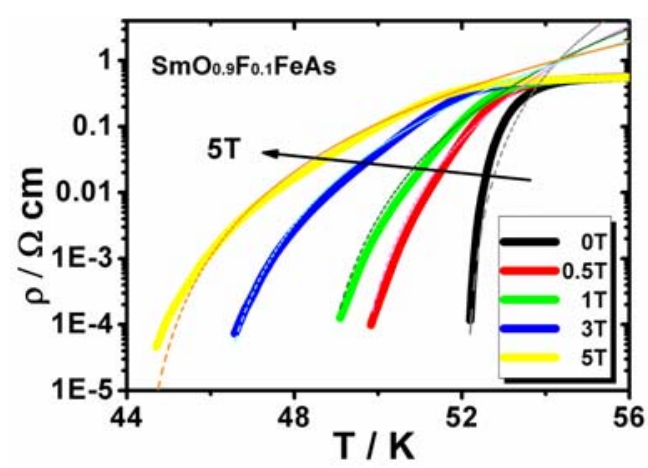

Fig. 1. The resistivity curves of $\mathrm{SmO}_{0.9} \mathrm{~F}_{0.1} \mathrm{FeAs}$ [16] as a function of temperature and magnetic field. The downward shift of $\mathrm{Tc}(\mathrm{B})$ in this sample is quite small. The dash line is a theoretical fitting with the parameters; $\rho_{n}=0.6 \mathrm{~m} \Omega \cdot \mathrm{cm}, T_{c}(0)=54 \mathrm{~K}$, and $v(z-1)=3.9 \pm 0.4$. 
Table 1. Summary of the results for the investigated samples. $\beta$ is obtained from the slope of the straight line fitting $1-T_{g}(B) /$ $T_{c}(0) \propto B^{\beta} . \beta_{S}$ is the slope of the straight line fitting $S+S_{0} \propto B^{\beta}$. Exponent $\lambda$ is obtained by plotting $\log (\mathrm{S})$ versus $\log (\mathrm{B})$ to compare it with the prediction of Tinkham. $v(z-1)$ is obtained by applying the Vogel-Fulcher relation.

\begin{tabular}{ccccc}
\hline \hline Sample & $v(z-1)$ & $\beta$ & $\beta s$ & $\lambda$ \\
\hline $\mathrm{SmO}_{0.9} \mathrm{~F}_{0.1} \mathrm{FeAs}^{[16]}$ & $3.9 \pm 0.4$ & 0.33 & 0.31 & 0.63 \\
$\mathrm{Y}_{0.8} \mathrm{Er}_{0.2} \mathrm{Ba}_{2} \mathrm{Cu}_{3} \mathrm{O}_{7-\mathrm{a}}{ }^{[17]}$ & $2.2 \pm 0.2$ & 0.61 & 0.58 & 0.71 \\
$\mathrm{MgB}_{2}{ }^{[18]}$ & $1.6 \pm 0.3$ & 0.89 & 0.81 & 0.86 \\
\hline
\end{tabular}

shows that the experimental data is in good agreement with our model.

A similar analysis has been performed for the other two samples. The main results are shown in Table 1. The local defect structure should have a profound influence on $v(z-$ 1), a possible explanation is the variation of the quenched disorder with different samples. A comparison between these three samples in Table 1 reveals that $\beta$ seems to decreases with increasing anisotropy. We should admit that equation $1-T_{g}(B) / T_{c}(0) \propto B^{\alpha}$ is a more logical expression because $T_{c}(0)$ is not equal to $T_{g}(0)$ in zero fields, and $1-T_{g}(B) / T_{c}(0) \propto B^{\beta}$ will be incorrect. So we combine these two scaling lines of glass temperature into a single figure (Fig. 2) to make a comparison and find that neither deviates from the power law distinctly, but our expression is more precise at temperature far from $T_{c}(0)$.

Let us continue to discuss Eqs. (6) which is the proof of the validity of Eqs. (2). Fig. 3 plots the numerical integration results of $\mathrm{SmO}_{0.9} \mathrm{~F}_{0.1} \mathrm{FeAs}$ and $\mathrm{Y}_{0.8} \mathrm{Er}_{0.2} \mathrm{Ba}_{2} \mathrm{Cu}_{3} \mathrm{O}_{7-\delta}$, whereas Table 1 shows the critical exponents. Firstly, we should be aware that $S_{0}$ must not be neglected since the symbols of $S_{0}$ are away from $S+S_{0}$ obviously. Our expression deviates from the integration results of $\mathrm{SmO}_{0.9} \mathrm{~F}_{0.1} \mathrm{FeAs}$ in the small field region and indicates that the glass behavior is weak since there are too few

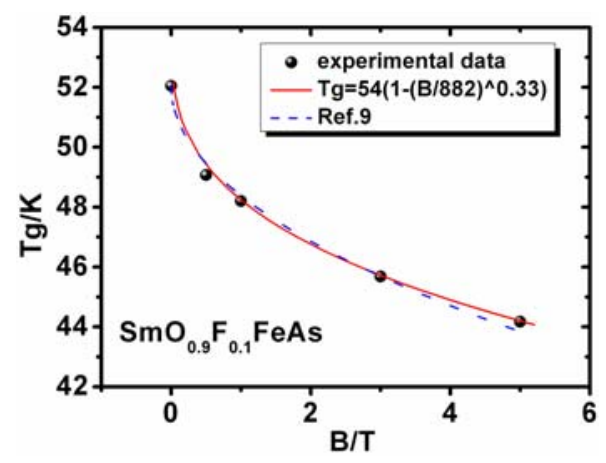

Fig. 2. The formula of $T_{g}$ vs. $B$ of $\mathrm{SmO}_{0.9} \mathrm{~F}_{0.1} \mathrm{FeAs}$ [16]. A comparison between our expression and $1-T_{g}(B) / T_{c}(0) \propto B^{\alpha}$ from Ref. 9 shows that our expression is more precise.
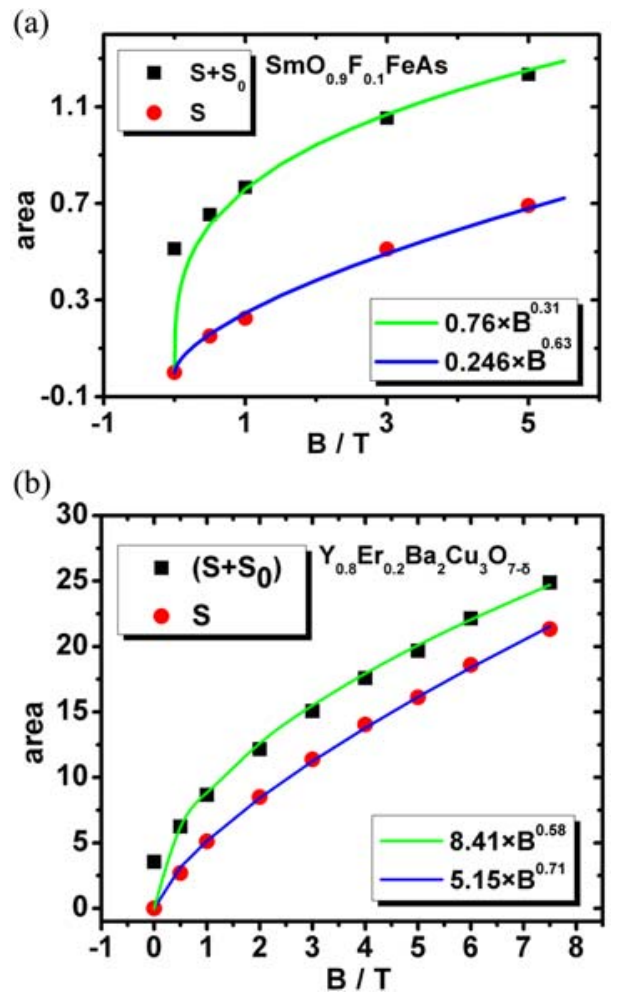

Fig. 3. The integral results of $\mathrm{SmO}_{0.9} \mathrm{~F}_{0.1} \mathrm{FeAs}$ [16] (a) and $\mathrm{Y}_{0.8} \mathrm{Er}_{0.2} \mathrm{Ba}_{2} \mathrm{Cu}_{3} \mathrm{O}_{7-\delta}$ [17] (b). The solid lines in (a) are theoretical fittings with the parameters; $\rho_{n}=0.6 \mathrm{~m} \Omega \cdot \mathrm{cm}, T_{c}(0)=54 \mathrm{~K}$, and $v(z-1)=4$ which is quite consistent with that in Fig. 1.

flux lines to exhibit glass behavior whereas the dissipation caused by the transport current at grain boundaries is relatively large in a near zero field. Secondly, comparing the exponent $\beta_{s}$ which is calculated from the numerical integration with the exponent $\beta$ obtained from $T_{g}$, we find that they are perfectly consistent with each other, which confirms the validity of Eqs. (6). The parameters obtained from the integral fitting coincide with the one from the $T_{g}$ fitting, as shown in the caption of Fig. 3. On the other hand, we also show the exponent $\lambda$ obtained by plotting $\log (S)$ versus $\log (B)$ in Table 1 to compare it with Tinkham's prediction of 0.67 . Our exponent qualitatively agreed with the prediction value and is consistent with other reports [19]. As has been reported [3,4], the properties of iron-based superconductors are similar with cuprate superconductors, but this is not the case in $\mathrm{MgB}_{2}$. Similar with Palstra's opinion [15], our explanation is that the weak-link networks are strong in complex layered compounds because the "vortex motion" belongs to an irreversible process in a disordered system whereas the alloy $\mathrm{MgB}_{2}$ does not have the layered structure. It is reasonable to observe this process only in a limited temperature range called the "irreversibility line" in HTSC. We should point out that our expression $S+S_{0} \propto B^{\beta}$ and 


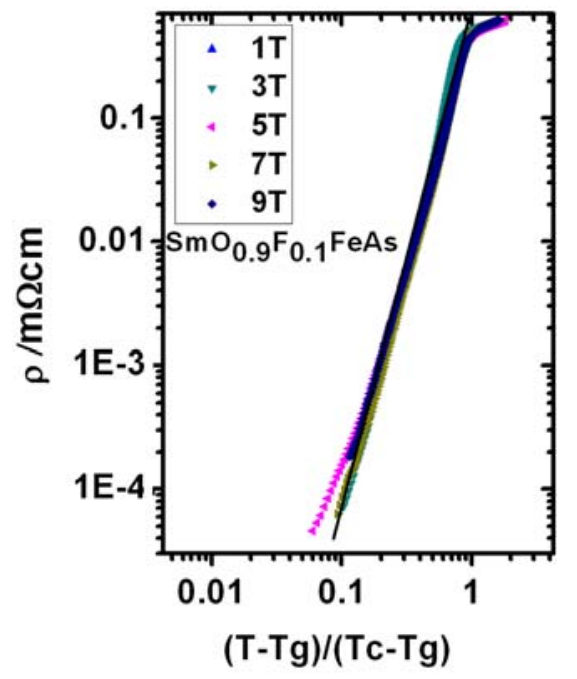

Fig. 4. Scaling of the temperature dependent resistance of $\mathrm{SmO}_{0.9} \mathrm{~F}_{0.1} \mathrm{FeAs}$ [16] under various magnetic fields. The slopes coincide with $v(z-1)$ from the result of Table 1 .

$S \propto B^{2 / 3}$ imply a more clear and simple relation between the vortex glass theory and "irreversibility line", which has been studied but is not clear in Palstra and Pankert [22]. Considering that $S$ and $S_{0}$ represent the total increase in dissipation caused by the field and current, respectively, the different mechanisms of dissipation may take an important role in these two behaviors and present different exponents.

Shown in Fig. 4 are the scaling results all of $\mathrm{SmO}_{0.9} \mathrm{~F}_{0.1^{-}}$ FeAs. The temperature dependent resistance in the vortex liquid region for various magnetic fields is scaled onto a straight line with a slope equal to $v(z-1)$ in Table 1 , therefore, this is more striking evidence for our model.

\section{Conclusion}

Our analysis uses the area of the magneto resistance vs. temperature curve. This method has the advantage of largely eliminating the contributions from sample imperfection and the complexity of channels available for electron transport to magnetic field dependence. A comparison between exponent $\beta$, which was calculated from our method and experiment data, confirms that our extended thermal activation model is a consistent description of the vortex glass theory. Furthermore, our model establishes a more clear relationship with Tinkham's model that means our model could also describe the "irreversibility line".

\section{Acknowledgment}

This work has been supported by NNSFC (10774065,
10523001) and NKPBRC (2006CB921802, 2010CB923404).

\section{References}

[1] Y. Kamihara, T. Watanabe, M. Hirano, and H. Hosono, J. Am. Chem. Soc. 130, 3296 (2008).

[2] Z. A. Ren, G. C. Che, X. L. Dong, J. Yang, W. Lu, W. Yi, X. L. Shen, Z. C. Li, L. L. Sun, F. Zhou, and Z. X. Zhao, Europhys. Lett. 83, 17002 (2008).

[3] H. H. Wen, G. Mu, L. Fang, H. Yang, and X. Zhu, Europhys. Lett. 82, 17009 (2008).

[4] C. de la Cruz, Q. Huang, J. W. Lynn, J. Y. Li, W. Ratcliff II, J. L. Zarestky, H. A. Mook, G. F. Chen, J. L. Luo, N. L. Wang, and P. C. Dai, Nature 453, 899 (2008).

[5] J. Dong, H. J. Zhang, G. Xu, Z. Li, G. Li, W. Z. Hu, D. Wu, G. F. Chen, X. Dai, J. L. Luo, Z. Fang, and N. L. Wang, Europhys. Lett. 83, 27006 (2008).

[6] A. Rydh, O. Rapp, and M. Anderson, Phys. Rev. Lett. 83, 1850 (1999).

[7] M. Anderson, A. Rydh, and O. Rapp, Phys. Rev. B. 63, 184511 (2001).

[8] S. L. Liu, G. J. Wu, X. B. Xu, J. Wu, and H. M. Shao, Supercond. Sci. Technol. 18, 1332 (2005).

[9] Y. Z. Zhang, Z. A. Ren, and Z. X. Zhao, Supercond. Sci. Technol. 22, 065012 (2009).

[10] M. P. A. Fisher, Phys. Rev. Lett. 62, 1415 (1989).

[11] R. H. Koch, V. Foglietti, W. J. Gallagher, G. Koren, A. Gupta, and M. P. A. Fisher, Phys. Rev. Lett. 63, 1511 (1989).

[12] D. S. Fisher, M. P. A. Fisher, and D. A. Huse, Phys. Rev. B 43, 130 (1991).

[13] D. A. Huse, M. P. A. Fisher, and D. S. Fisher, Nature 358, 553 (1992).

[14] M. Tinkham, Phys. Rev. Lett. 61, 1658 (1988).

[15] T. T. M. Palstra, B. Batlogg, R. B. van Dover, L. F. Schneemeyer, and J. V. Waszczak, Phys. Rev. B 41, 6621 (1990).

[16] Z. A. Ren, W. Lu, J. Yang, W. Yi, X. L. Shen, Z. C. Li, G. C. Che, X. L. Dong, L. L. Sun, F. Zhou, and Z. X. Zhao, Chin. Phys. Lett. 25, 2215 (2008).

[17] Z. H. Wang, X. W. Zou, J. Fang, T. Yang, Y. L. Tang, Z. Huang, L. Qiu, J. L. Chen, H. Zhang, and S. Y. Ding, Supercond. Sci. Technol. 15, 183 (2002).

[18] S. Patnaik, A. Gruevich, S. D. Bu, S. D. Kaushik, J. Choi, C. B. Eom, and D. C. Larbalestier, Phys. Rev. B 70, 064503 (2004).

[19] V. V. Gridin, T. W. Krause, P. K. Ummat, and W. R. Datars, Solid State Commun. 78, 515 (1991).

[20] L. F. Hou, J. Deak, P. Metealf, M. McElfresh, and G. Preosti, Phys. Rev. B 55, 11806 (1997).

[21] H. Safar, P. L. Gammel, D. J. Bishop, D. B. Mitzi, and A. Kapitulnik, Phys. Rev. Lett. 68, 2672 (1992).

[22] J. Pankert, G. Marback, A. Comberg, P. Lemmerns, P. Froning, and C. Ewert, Phys. Rev. Lett. 65, 3052 (1990). 